MATEC Web of Conferences 22,02016 (2015)

DOI: $10.1051 /$ matecconf/ 20152202016

(C) Owned by the authors, published by EDP Sciences, 2015

\title{
Low-Carbon Economic Dispatching for Power Grid Integrated with Car- bon Capture Power Plants and Wind Power System
}

\author{
Siqing Sheng, Liangliang $\mathrm{Li}^{*} \&$ Meng Liu \\ Electrical and Electronic Engineering Institute, North China Electric Power University, Baoding, Hebei, China
}

\begin{abstract}
Carbon emission characteristics of all kinds of power units are analyzed against the background of the low carbon economy. This paper introduces carbon trading in the dispatching model, gives full consideration to the benefit or cost of carbon emission and introduces carbon emission in the dispatching model as a decision variable so as to achieve the unity of the economy and the environmental protection of the dispatching model. A low carbon economic dispatching model is established based on multiple objectives, such as the lowest thermal power generation cost, the lowest carbon trading cost and the lowest carbon capture power plant operation cost. Load equalization, output constraint of power unit, ramping constraint, spinning reserve constraint and carbon capture efficiency constraint should be taken into account in terms of constraint conditions. The model is solved by the particle swarm optimization based on dynamic exchange and density distance. The fact that the introduction of carbon trading can effectively reduce the level of carbon emission and increase the acceptance level of wind power is highlighted through the comparison of the results of three models' computational examples. With the carbon trading mechanism, carbon capture power plants with new technologies are able to give full play to the advantage of reducing carbon emission and wind curtailment so as to promote the development of the energy conservation and emission reduction technology and reduce the total cost of the dispatching system.
\end{abstract}

Keywords: carbon trading; carbon capture power plant; wind power generation; low carbon economic dispatch

\section{INTRODUCTION}

With the development of the economy, the issue of global climate change draws people's attention. It has become a consensus that global warming should be coped with by the reduction of $\mathrm{CO}_{2}$ emission ${ }^{[1]}$. Coal generation is the main structure of energy generation in China and thermal power generation accounts for more than $70 \%$ of China's power generation structure [2]. This pattern of energy generation cannot be changed in a short period of time in the future. The power industry has much to offer by developing a low carbon economy and realizing the sustainable development of the economy.

Despite the merit of being clean and pollution-free, wind power generation has the defect of being intermittent and volatile. This also restricts the connection of wind power in the power grid. People have made a large number of studies on the grid connection of wind power. In Literature [3], the penalty cost of wind power is introduced in the objective function according to the defect of wind power of being intermittent and volatile, which takes into account the economic efficiency of dispatching as well as the maximum wind power acceptance. Literature [4] studies the influencing factors for the dynamic economy dispatching of the electric power system with wind power plants and calculates the energy consumption cost brought by the valve point effect of the conventional thermal power unit through objective functions. The environmental protection benefit of wind power has

*Corresponding author: liliangliang412@163.com drawn more and more attentions against the background of the low carbon economy. Objective functions regarding to power generation cost and carbon emission are established in Literature [5], which adds the risk cost in the power generation cost function according to the defect of wind power and uses the improved multi-objective particle swarm optimization for solution. But the literature only takes the minimum carbon emission into account instead of the economic benefits brought by carbon emission. Literature [6] puts forward a multi-objective optimization model of the low carbon economy with wind power system based on scenario probability, takes into account the influence of carbon trading, proposes such indicators as average power purchase cost, wind power price subsidy and average output share of units, and verifies the feasibility of the model. Advantages of the carbon capture power plant have drawn people's attention against the background of the low carbon economy. In Literature [7], advantages of the carbon capture power plant are introduced from the aspect of power plant and power system. It reveals the research emphasis and the development direction of the flexible operation mode of the carbon capture power plant. Literature [8] mainly introduces advantages of the carbon capture power plant in terms of peak regulating of power system and evaluates its peak regulating mechanism and benefit. In Literature [9], the carbon capture power plant and the virtual electrical changing station are introduced in the dispatching model against the background of the carbon market. But it only consid- 
ers the low carbon benefit of the carbon capture power plant, with no regard to other advantages.

By introducing carbon trading, this paper not only establishes a dispatching model with such objective functions as the lowest thermal power generation cost, the lowest carbon trading cost and the lowest carbon capture power plant operation cost but also obtains the optimal solution of the model through the improved particle swarm optimization. It is highlighted through the comparison of three dispatching models that the introduction of carbon trading can effectively reduce the level of carbon emission, increase the acceptance level of wind power and give full play to the advantages of the carbon capture power plant so as to the total cost of the dispatching system and realize the unity of economy and low carbon of the dispatching model.

\section{ESTABLISHMENT OF A LOW CARBON ECONOMY DISPATCHING MODEL}

\subsection{An analysis on features of the carbon emission of normal power supply}

$\mathrm{CO}_{2}$ emission of renewable generation modes like nuclear power, wind power and water power is almost zero. So the carbon emission can be neglected.

The carbon emission of a conventional thermal power unit is related to parameters of the unit, which can be calculated through the following formula:

$$
F_{1 i, t}=\frac{11}{3} \xi_{i} P_{G i, t} \Delta t \delta K_{c o_{2}}
$$

Where, $11 / 3$ is the molar mass ratio of $\mathrm{CO}_{2}$ and $\mathrm{C}$; $P_{G i, t}$ is the generated output of the unit $i$ in the time period $\mathrm{t} ; \xi_{i}$ is the standard coal consumption for power generation of the unit $i ; \delta$ is the carbon content of standard coal; ${ }^{\mathrm{CO}_{2}}$ is the oxidation rate of carbon.

\subsection{Carbon emission features of the carbon capture power plant}

Carbon capture technology is also called carbon capture and storage (CCS), including such links as CO2 capture, transportation, storage, and so on. It generally reduces the carbon emission of power generation per unit by $85 \% \sim 90 \%{ }^{[10]}$. Based on the original thermal power unit, the carbon capture power plant is equipped with a carbon capture system to capture $\mathrm{CO} 2$ with a certain consumption of generated output so as to reduce carbon emission of the thermal power unit significantly. The carbon emission of the carbon capture power plant can be calculated with the following formula:

$$
F_{2 i, t}=\frac{11}{3} \xi_{i} P_{G i, t} \Delta t \delta K_{c o_{2}}\left(1-\eta_{i, t}\right)
$$

Where, @ is the carbon capture efficiency of the unit $i$ in the time period $t$.

\subsection{Introduction of carbon trading}

Carbon trading is a trading mechanism that realizes carbon emission by establishing a legal right for carbon emission and allows the business of this right. With the principle of controlling the total carbon emission, the government assigns the carbon emission right to emission sources. When the actual emission of a source is smaller than the assigned emission allowance, the remaining allowance can be sold in the market to make a profit. When the actual emission of a source is larger than the assigned emission allowance, the extra part should be purchased in the market. Otherwise the emission source will be punished with a heavy fine ${ }^{[11]}$.

The distribution mode of the initial free carbon emission right in accordance with generating capacity is adopted in this paper. The distribution mode that carbon emission allowance is directly proportional to generating capacity is applied to different generating units. The assigned carbon emission allowance of each unit is:

$$
\pi_{i}=\frac{P_{N i}}{\sum_{i=1}^{N_{1}+N_{2}} P_{N i}} \times \frac{P_{L, t}}{\sum_{t=1}^{T} P_{L, t}} E_{q}
$$

Where, $E$ is the total allowance of carbon emission; $P_{L, t}$ is the total load in the time period $t ; P_{N i}$ is the installed capacity of the unit $i ; N_{1}$ and $N_{2}$ are respectively the unit installed with a carbon capture device and the conventional thermal power unit.

\subsection{Establishment of the low carbon dispatching model based on carbon trading}

The operational cost of the thermal power unit is the minimum in the objective function 1:

$$
\min f_{1}=\sum_{t=1}^{T} \sum_{i=1}^{N}\left(a_{1 i}+b_{1 i} p_{i, t}+c_{1 i} p_{i, t}^{2}\right)
$$

The cost of carbon trading is the minimum is the objective function 2 :

$\min f_{2}=P_{c 0_{2}}\left\{\sum_{i=1}^{N_{1}}\left(\sum_{t=1}^{T} F_{i, t}-E_{i, t}-\pi_{i}\right)+\sum_{i=N_{1}+1}^{N_{2}}\left(\sum_{t=1}^{T} F_{i, t}-\pi_{i}\right)\right\}$

The country should offer carbon capture power plants with certain subsidies in line with relevant policies in consideration of the fact that the operation of a carbon capture power plant needs to consume a certain amount of generated power. The operational cost of the carbon capture power plant is the minimum in the objective function 3 :

$$
\min f_{3}=\sum_{t=1}^{T} \sum_{i=1}^{N_{1}}\left(a_{2 i} P_{C i, t}+b_{2 i}\right)-\zeta \sum_{t=1}^{T} \sum_{i=1}^{N_{1}} E_{i, t}
$$

Where, $T$ is a dispatching cycle; $N$ is the total number of thermal power units that can be dispatched; $a_{1 i}, b_{2 i}$ and $c_{1 i}$ are respectively coal cost coefficients of the $i$ thermal power unit; $a_{\eta_{i}}$ and $b_{\gamma_{i}}$ are 
operational cost coefficients of the carbon capture power plant; ${ }^{P} C i, t$ is the operational power of the carbon capture power plant. The calculation formula is:

$P_{C i, t}=\frac{11}{3} \alpha_{i} \xi_{i} P_{G i, t} \delta K_{c o_{2}} \eta_{i, t}+\beta_{i}$

Where, $\alpha_{i}$ is the $\mathrm{CO}_{2}$ capture consumption per unit of the carbon capture system of the unit $i ; \beta_{i}$ is the basic operational consumption of the carbon capture system of the unit $i ; \zeta$ is the subsidy for carbon capture power plants, yuan $/ t ; P_{c o_{2}}$ is the price of carbon trading, yuan $/$.

\subsection{Constraint conditions}

(1)Power balance constraint of the system:

$$
\sum_{i=1}^{N} P_{s i, t}+P_{w, t}=P_{L, t}
$$

Where, $P_{s i, t}$ is the on-grid power of the unit $i$ in the time period of $t$; the on-grid power of a conventional thermal power unit is the unit output; the on-grid power of a carbon capture power plant is the difference value between the unit output and the operational power of the carbon capture power plant; $P_{P, t}$ is the wind power output in the time period of $t ; P_{L, t}^{w, t}$ is the load requirement in the time period of $t$.

(2) Output constraint of the generator unit:

$\left\{\begin{array}{l}P_{G i}^{\min } \leq P_{G i, t} \leq P_{G i}^{\max } \\ 0 \leq P_{W, t} \leq P_{W, t}^{\max }\end{array}\right.$

Where, $P_{G i}^{\max }$ and $P_{G i}^{\min }$ are the maximum and the minimum values of the unit $i$ in the time period of $t ; P_{W, t}^{\max }$ is the maximum output of the wind power generator unit $i$ in the time period of $t$.

(3) Ramping rate constraint of the unit:

$\left\{\begin{array}{l}P_{G i, t}-P_{G i, t-1} \leq U_{G i} \\ P_{G i, t-1}-P_{G i, t} \leq Z_{G i}\end{array}\right.$

Where, $U_{G i}$ and $Z_{G i}$ are respectively limiting values of the increasing and decreasing rates of the unit $i$

(4)Efficiency constraint of the carbon capture system:

$$
0 \leq \eta_{i, t} \leq \eta_{i}^{\max }
$$

Where, @ is the maximum carbon capture rate of a carbon capture power plant.

(5) Spinning reserve constraint of the system:

$$
\left\{\begin{array}{l}
\sum_{i=1}^{N_{1}+N_{2}} P_{S i, t}^{\max }-\sum_{i=1}^{N_{1}+N_{2}} P_{S i, t} \geq u_{1} P_{L, t}+u_{w} P_{W, t}^{\max } \\
\sum_{i=1}^{N_{1}+N_{2}} P_{S i, t}-\sum_{i=1}^{N_{1}+N_{2}} P_{S i, t}^{\max } \geq d_{1} P_{L, t}+d_{w} P_{w, t}^{\max }
\end{array}\right.
$$

Where, $u_{1}, d_{1}$ and $u_{w}, d_{w}$ are respectively positive and negative spinning reverse caused by errors of the load forecasting and the wind power forecasting.

\section{ALGORITHM OF MODEL SOLUTION}

As for the multi-objective optimization problem of this paper, the multi-objective particle swarm optimization based on dynamic exchange and density distance is applied to solving non-dominant sets and the optimal particle is then searched in the non-dominant set as the optimal solution of the model.

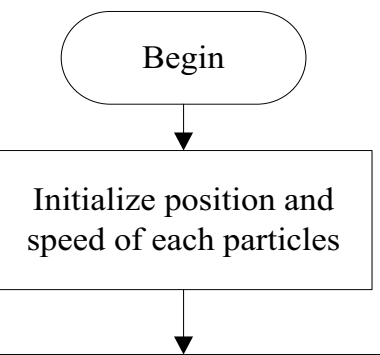

Based on the concept of fitness, dividing the initial population into dominant and non dominant sets

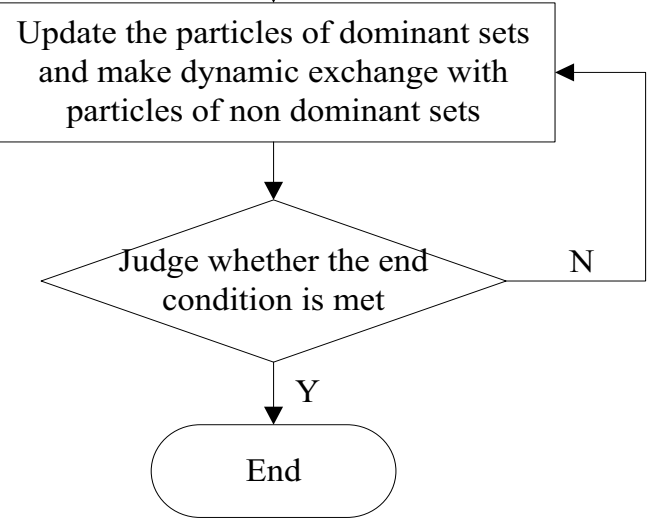

Figure 1. The procedure of the algorithm

The dynamic exchange process is that a particle swarm is divided into a dominant set and a non-dominant set with the concept of fitness dominance and only the speed and the position of particles in the dominant set are updated each time. Exchange excellent particles in the dominant set with particles in 


\section{MATEC Web of Conferences}

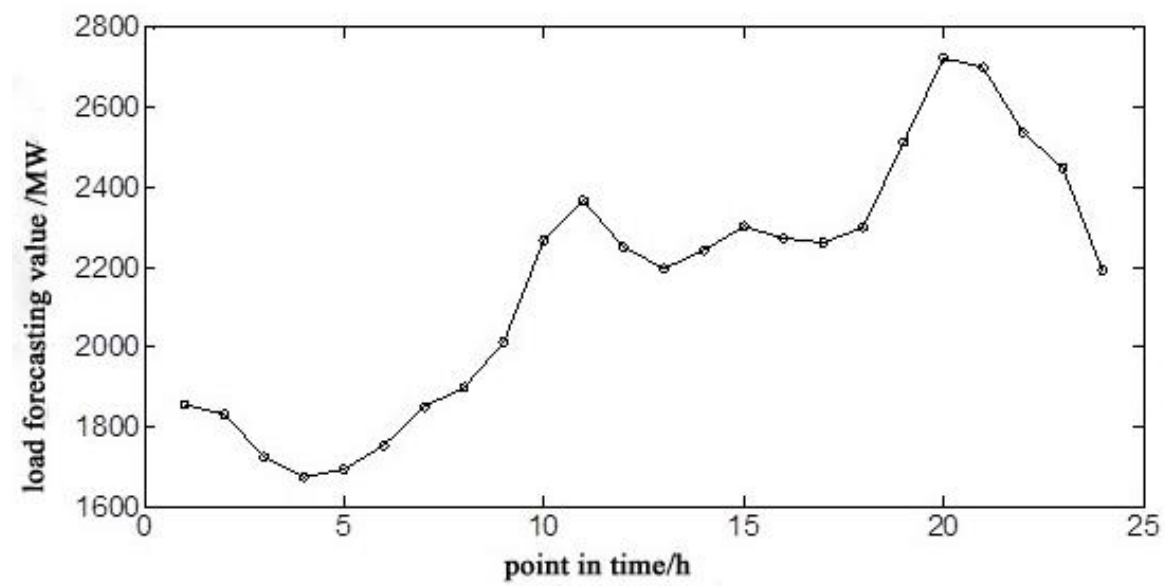

Figure 2. Load forecasting

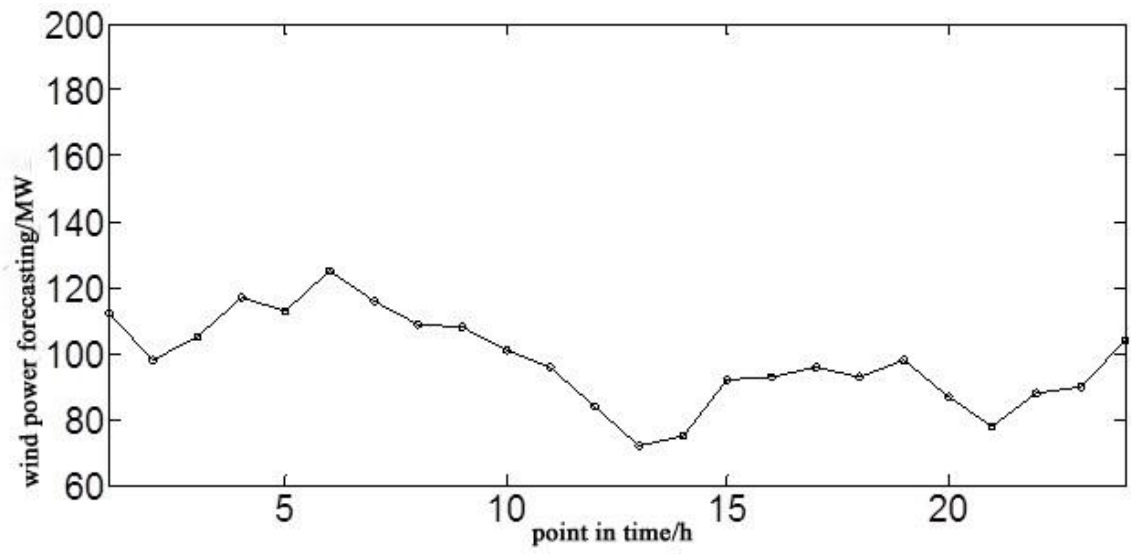

Figure 3. Wind power forecasting

the non-dominant set so as to guarantee that particles in the non-dominant set are close to the Proteto edge. Refer to Literature [12] for the specific dynamic exchange process.

In order to avoid the fact that the particle swarm optimization is trapped in the local optimum prematurely and increase the optimizing ability of the algorithm, the particle with the largest density distance in the non-dominant set is selected as the global optimal solution of the velocity updating. The density distance can be expressed as ${ }^{[12]}$ :

$C_{i}=\frac{d_{i}^{1}+d_{i}^{2}}{2}$

Where, $d_{i}^{1}$ and $d_{i}^{2}$ are the smallest two Euclidean distances between particle $i$ and other particles in the non-dominant set; $C_{i}$ is the density of other indi- viduals around particle $i$.

The procedure of the algorithm is provided in figure 1.

\section{CALCULATION EXAMPLE ANALYSIS}

\subsection{System testing}

The dispatching system in this paper includes five thermal power units and a wind generating unit with an installed capacity of $130 \mathrm{MW}$. Unit 1 and unit 2 are equipped with the carbon capture system. 24 time periods in a day are dispatching cycles. Refer to Literature [9] for specific parameters and constraint conditions of the units. The carbon trading price and the subsidized price of the nation for carbon capture power plants are respectively $120 y u a n / t$ and $150 y u a n / t$. The total allowance of carbon emission is 42000 . The load forecasting output and the wind power forecast- 
ICETA 2015

Table 1. Dispatching results of each unit

\begin{tabular}{|c|c|c|c|c|c|c|c|c|c|c|c|c|c|}
\hline $\begin{array}{l}\text { Time } \\
\text { period }\end{array}$ & $P_{G 1}$ & $P_{G 2}$ & $P_{G 3}$ & $P_{G 4}$ & $P_{G 5}$ & $P_{W}$ & $\begin{array}{l}\text { Time } \\
\text { period }\end{array}$ & $P_{G 1}$ & $P_{G 2}$ & $P_{G 3}$ & $P_{G 4}$ & $P_{G 5}$ & $P_{W}$ \\
\hline 1 & 475.72 & 370.43 & 588.84 & 424.38 & 425.07 & 111.56 & 13 & 608.47 & 425.00 & 450.77 & 600.00 & 310.00 & 71.23 \\
\hline 2 & 450.92 & 395.26 & 400.34 & 355.46 & 310.00 & 96.59 & 14 & 610.00 & 425.00 & 525.41 & 598.49 & 310.00 & 74.87 \\
\hline 3 & 460.42 & 316.76 & 350.23 & 325.81 & 319.84 & 104.59 & 15 & 608.47 & 425.00 & 350.00 & 547.60 & 516.64 & 91.56 \\
\hline 4 & 312.48 & 256.67 & 350.00 & 325.00 & 310.00 & 116.42 & 16 & 510.72 & 423.93 & 605.15 & 355.28 & 513.57 & 83.01 \\
\hline 5 & 319.95 & 259.37 & 350.00 & 325.00 & 311.43 & 112.45 & 17 & 459.23 & 420.84 & 649.69 & 429.48 & 468.11 & 75.98 \\
\hline 6 & 304.63 & 265.04 & 350.00 & 325.00 & 310.16 & 123.45 & 18 & 559.24 & 334.12 & 529.41 & 598.49 & 397.80 & 82.59 \\
\hline 7 & 389.49 & 280.15 & 351.98 & 363.52 & 515.47 & 115.98 & 19 & 582.37 & 335.05 & 606.82 & 600.00 & 570.00 & 97.64 \\
\hline 8 & 530.85 & 423.93 & 350.72 & 325.81 & 310.77 & 108.62 & 20 & 610.00 & 381.36 & 700.00 & 600.00 & 570.00 & 86.82 \\
\hline 9 & 430.52 & 352.52 & 350.00 & 513.53 & 455.56 & 107.89 & 21 & 608.98 & 423.61 & 700.00 & 600.00 & 570.00 & 77.97 \\
\hline 10 & 610.00 & 408.06 & 376.30 & 506.44 & 480.30 & 100.85 & 22 & 608.47 & 383.70 & 700.00 & 600.00 & 453.34 & 87.63 \\
\hline 11 & 586.12 & 351.07 & 676.30 & 373.03 & 484.30 & 96.08 & 23 & 565.80 & 328.51 & 619.47 & 598.49 & 513.40 & 89.56 \\
\hline 12 & 608.47 & 425.00 & 473.71 & 600.00 & 310.00 & 83.85 & 24 & 608.47 & 425.00 & 350.00 & 381.97 & 568.57 & 104.00 \\
\hline
\end{tabular}

ing output are shown in Figure 2 and Figure 3.

\subsection{Results of low carbon dispatching}

It can be known from the dispatching data that there is no carbon trading cost because wind power generation has no emission of greenhouse gases. So, wind power can basically realize the connection to the grid with full allowance. The carbon capture power plant (unit 1 and unit 2) is able to reduce the carbon emission of units, so unit 1 and unit 2 can maintain a relatively high level of output. Based on the fact that constraint conditions of the model are satisfied, the dispatching scheme of this paper utilizes wind power and carbon capture power plants to a larger extent and realizes the balance between the low carbon dispatching and the economic dispatching.

\subsection{Comparison of different dispatching models}

Table 2. Results comparison of different models

\begin{tabular}{|l|l|l|l|}
\hline Models & 1 & 2 & 3 \\
\hline$E_{p} /$ million ton & 4.25 & 4.16 & 4.01 \\
\hline$f_{1} /$ million ton & 2016 & 2026 & 2039 \\
\hline$f_{2} /$ million ton & 6.01 & -4.83 & -22.52 \\
\hline$f_{3} /$ million ton & - & - & 1.71 \\
\hline $\begin{array}{l}\left(f_{1}+f_{2}+f_{3}\right) / \text { million } \\
\text { ton }\end{array}$ & 2022.01 & 2021.17 & 2018.19 \\
\hline
\end{tabular}

In order to highlight the effect of carbon trading and carbon capture power plant, three kinds of dispatching models are compared in this section. Model 1: Apply the traditional dispatching model, which takes the minimum operational cost $f_{1}$ of the thermal power unit as the objective function and shut down the carbon capture system. Model 2: Shut down the carbon capture system of this model without regard to the low carbon dispatching model of carbon trading under the operational condition of the carbon capture system. Model 3 (the model in this paper): solve various costs of the system in consideration of the carbon trading situation under the operational condition of the carbon capture system.

As shown in Table 2, the objective function in
Model 1 only pursues the maximization of economic benefit. Although the thermal power generation cost is lowest, the carbon emission is the highest. The carbon trading cost as high as 60100 yuan should be paid. The total cost of Model 1 is the highest. The carbon emission of Model 2 is reduced by $2.11 \%$ compared with Model 1 due to the introduction of carbon trading. A benefit of 48300 yuan can be obtained in the carbon trading. This is because both the economic benefit and the carbon emission of a unit should be considered in the system dispatching. The total cost of Model 2 is between Model 1 and Model 3. The carbon emission of Model 3 is reduced to 4.01 million tons due to the utilization of the carbon capture system, which is reduced by $5.64 \%$ compared with Model 1 and brings a carbon trading benefit of 225200 yuan. However, the thermal power operation cost of Model 3 is increased because the start of the carbon capture system consumes a certain amount of generated power. The government gives appropriate subsidies according to the carbon capture amount of carbon capture power plants in order to popularize the carbon capture technology. Model 3 still needs to pay a cost of 17100 yuan in spite of its subsidy. But the cost accounts for only 7.6\% of the carbon trading profits. The total cost of Model 3 is the lowest. Besides, it can be also found that public subsidies are necessary at the early stage of popularizing the carbon capture power plant. Without the public subsidy, Model 3 needs to pay an operating cost of 82100 yuan for the carbon capture power plant. This will increase the cost of Model 3 and weakens the role of carbon trading, which is to the disadvantage of the popularization of the carbon capture technology and the carbon trading mechanism.

\subsection{Influence of carbon capture power plant on Model 3}

Wind power has the feature of being anti-peak-regulation, which widens the difference between the peak and the valley of the load. The wind power output is large when the load demand is small and the wind power output is small when the load demand is large. The carbon capture power plant is 


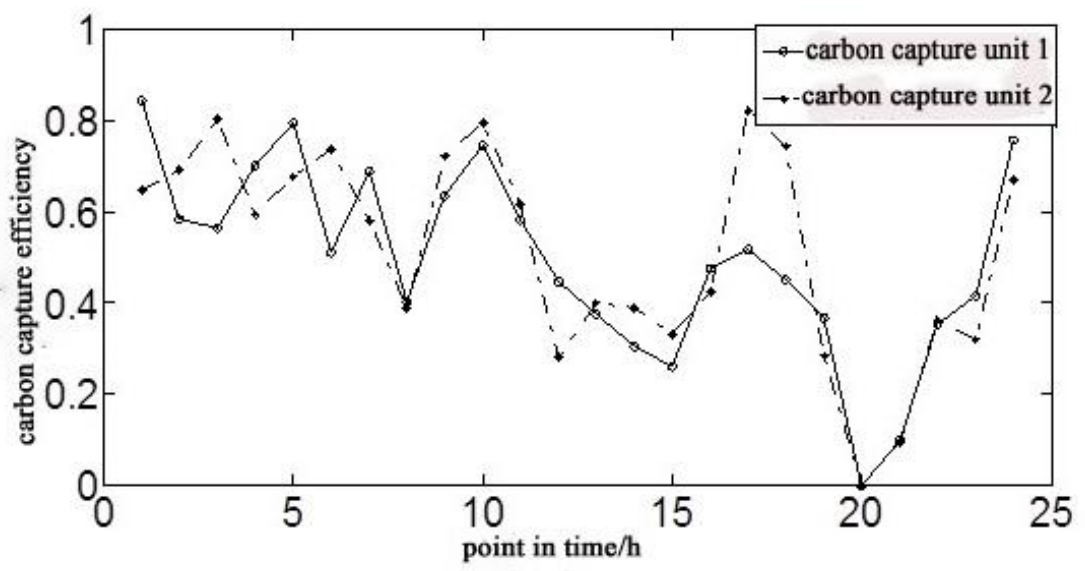

Figure 4. Carbon capture efficiency

able to improve the efficiency of carbon capture and reduce the on-grid power of a unit when the net load of the system is relatively small. The carbon capture power plant is able to decrease the efficiency of carbon capture, increase the on-grid power of the system and compensate for the insufficient output of thermal power units when the net load is relatively large. The change of the carbon capture efficiency over time in the dispatching Model 3 is shown in Figure 4.

A carbon capture power plant is connected to the system in Model 3. On the one hand, the connection of wind power reduces the output of thermal power units, maintains a high level of the carbon capture efficiency and reduces the carbon emission of the system; on the other hand, the carbon capture efficiency maintains a high level and the output of thermal power units and the wind curtailment amount of the system are reduced when the net load is in the valley. The time period between 3 and 6 is the valley of the load. Units are operating around the lower limit of output. But units in Model 2 are still unable to satisfy the load balance and only have the choice of wind curtailment, the total amount of which is $75.24 \mathrm{MW}$. Model 3 can basically realize the full connection of wind power by improving the carbon capture efficiency, reducing the lower output limit of units and increasing the on-grid amount of wind power.

\section{CONCLUSION}

In the background of low carbon economy development, this paper introduces carbon trading in objective function and establishes a low carbon economic dispatching model with such multiple objectives as the lowest thermal power generation cost, the lowest carbon trading cost and the lowest carbon capture power plant operation cost. The dispatching unit contains a carbon capture power plant, a thermal power unit and a wind power plant. The establishment of the model takes fully into account the unity of the economy and the environmental protection of the dispatching model. The multi-objective model is solved with the particle swarm optimization based on dynamic exchange and density distance. It can be concluded from the comparison of three dispatching models that the introduction of carbon trading and carbon capture power plant can effectively reduce carbon emission of the dispatching model. The carbon capture power plant can effectively reduce carbon emission and wind curtailment of wind power and increase the level of wind power acceptance. The carbon capture power plant and the carbon trading mechanism have a bright future in the development process of low-carbon electricity.

\section{REFERENCES}

[1] IPCC. 2005. Special Report on Carbon Dioxide Capture and Storage, Cambridge, UK: Cambridge University Press.

[2] Chen, Q.X., Kang, C.S. \& Xia, Q., et al. 2009. An analysis on key factors of the low carbonization of the power industry and the influence on power source planning, Automation of Electric Power Systems, 33(15): 18-23.

[3] Dong, X.T., Yan, Z. \& Feng, D.H., et al. 2012. Economic dispatching of the power system involving wind power output penalty cost, Power System Technology, (8): 76-80.

[4] Zhou, W., Peng, Y. \& Sun, H. 2009. Economic dispatching of the power system with wind power plant, Proceedings of the CSEE, 29(25): 13-18.

[5] Zhang, X.H. \& Dong, X.H. 2013. A study on the dynamic economic dispatching of the multi-objective low-carbon electric power system with wind power plant, Power System Technology, pp: 25-31.

[6] Zhang, X.H., Yan, K.K., Lu, Z.G. \& Zhong, J.Q. 2014. Low-carbon economic dispatching of the electric power 
system based on scenario probability, Power System Technology, 78(7): 1836-1841.

[7] Kang, C.Q., Ji, Z. \& Chen, Q.X. 2012. A review and the outlook of flexible operational methods of carbon capture power plant, Automation of Electric Power Systems, (3): 1-9.

[8] Chen, Q.X., Kang, C.Q., \& Xia, Q. 2010. A study on the operational mechanism and peak-regulation efficiency of carbon capture power plant, Proceedings of the CSEE, 30(7): 22-28.

[9] Gao, Y.J., Li, R.H., Liang, H.F. \& Zhang, J.C. 2014. The optimization dispatching of electric power system involving carbon capture power plant and electrical changing station in the carbon market environment, $\mathrm{Au}$ tomation of Electric Power Systems, 38(17): 150-156.

[10]IPCC. 2005. Special Report on Carbon Dioxide Capture and Storage, Cambridge: Cambridge University Press, pp: 2-4.

[11]Wang, X. \& Yuan, F. 2010. Emission rights distribution and price management in carbon emissions permit trading, Financial Development Review, (11): 48-57.

[12]Jin, X.L. 2006. A Study on the Multi-objective Optimization Algorithm Based on PSO and its Application, Hangzhou: Zhejiang University. 